\author{
M.V. TKACH, YU.O. SETI \\ Yu. Fed'kovich National University of Chernivtsi \\ (2, Kotsyubyns'kyi Str., Chernivtsi 58012, Ukraine; e-mail: ktf@chnu.edu.ua)
}

\title{
THEORY OF THE PROPERTIES
}

OF RESONANT-TUNNELING NANOSTRUCTURES

PACS 73.21.Fg, 73.90.+f,

72.30. $+\mathrm{q}, 73.63 . \mathrm{Hs}$

\section{AS ACTIVE ELEMENTS OF QUANTUM CASCADE LASERS AND DETECTORS}

\begin{abstract}
A theory describing the spectral parameters of quasistationary states and the dynamic conductivity in an open three-barrier resonant-tunneling system (RTS) as an active element of a quantum cascade laser or a quantum cascade detector has been developed in the framework of the rectangular potential model and the effective electron mass approximation. It is shown that the optimal functioning of a three-barrier RTS can be obtained by properly choosing its geometric configuration, namely, by arranging the inner barrier closer to the input one in a position determined by the electromagnetic field energy.

Keywords: resonance-tunnel nanostructure, conductivity
\end{abstract}

\section{Introduction}

For the last decade, quantum cascade lasers (QCLs) and quantum cascade detectors (QCDs) have been intensively researched and developed, which comprises one of important directions in nanotechnologies. Among the reasons for why such attention is paid to those researches is the fact that the devices concerned operate in the interesting terahertz range of electromagnetic waves, which contains the so-called atmospheric "transparency windows". Such advantages as small dimensions, stable functioning at high temperatures, consumption of low currents, and highprecision characteristics distinguish nano-QCLs and nano-QCDs among the devices of other types.

The difficulties associated with QCLs and QCDs consist in that the experimental or technological fabrication of those devices demands an expensive work of high-precision installations, e.g., those for molecular beam epitaxy. The theoretical difficulties are associated with the necessity to study physical phenomena in open RTSs, which are active elements of QCLs and QCDs.

The principle of operation of a nanolaser based on quantum transitions between quasistationary electron states (QSESs) in plane resonant-tunneling structures was proposed for the first time in work [1] in 1972 and consisted in the following. An electron emitted by an injector (from the left) tunnels in the

(C) M.V. TKACH, YU.O. SETI, 2013

182
RTS onto an excited QSES of dimensional quantization, emits a quantum of electromagnetic energy, and transits onto a lower or the ground QSES to tunnel from it through the RTS (to the right).

The first really functioning nanolasers were created only in 1994-1995 by J. Faist, F. Capasso et al. [2,3].

The main principles of operation of really functioning QCLs and QCDs $[4,5]$ are as follows. A quantumwell QCL (QWCL) contains several tens of identical cascades. Every of them conditionally consists of an injector and an active zone. The latter is composed of several nano-sized plane layers of different materials that form energy wells and barriers; for example, GaAs and AlAs. Electrons from the injector get onto an excited QSES, emit a quantum of the electromagnetic field, transit into a lower QSES, and tunnel into the next injector. Further, the process runs similarly, because the electron so relaxes in the injector that its energy, when it arrives at the next cascade, should be the same as in the former one.

The work of a QCD is based on that electrons from the injector are directed onto the ground level of RTS, absorb quanta of the electromagnetic field, transit onto one of higher QSESs, tunnel into the extractor, relax there, and get onto the ground QSES in the next cascade. The first high-quality QCD was created in 2001 [6] and was named the quantum-well infrared photodetector (QWIP), since it functions in the mid-infrared frequency range.

It should be noticed that, owing to the absence of a consistent theory of open nanosystems, the ba-

ISSN 2071-0194. Ukr. J. Phys. 2013. Vol. 58, No. 2 
sic estimations in experimental works were carried out on the basis of the theory for closed nanostructures. It enabled the energy spectrum to be reasonably estimated, but did not allow the active conductivity of RTS, as an essentially open system, to be calculated. A development of a consistent theory of the electron conductivity in an open three-barrier resonant-tunneling structure (3BRTS) in the framework of rather a simple model of rectangular potential wells and barriers with different effective masses of electron in them comprises the main purpose of this work.

\section{Theory of Active Conductivity in a Three-Barrier Resonant-Tunneling Structure with Shifted Well Bottoms}

An open planar 3BRTS with shifted depths of potential wells is considered (Fig. 1). The axis $z$ of the Cartesian coordinate system is directed perpendicularly to nanoheterosystem's planes. An insignificant difference between the lattice constants for layerswells and layers-barriers of 3BRTS allows us to use the model of effective masses,

$$
\begin{aligned}
& m(z)=m_{0} \theta(-z)+m_{1} \sum_{i=0}^{2}\left(\theta\left(z-z_{2 i}\right)-\theta\left(z-z_{2 i+1}\right)\right)+ \\
& +m_{2} \sum_{i=1}^{3}\left(\theta\left(z-z_{2 i-1}\right)-\theta\left(z-z_{2 i}\right)\right)
\end{aligned}
$$

and rectangular potential barriers,

$$
\begin{aligned}
& U(z)=U \sum_{i=0}^{2}\left(\theta\left(z-z_{2 i}\right)-\theta\left(z-z_{2 i+1}\right)\right)=- \\
& -V \sum_{i=1}^{3}\left(\theta\left(z-z_{2 i-1}\right)-\theta\left(z-z_{2 i}\right)\right) .
\end{aligned}
$$

Here, $\theta(z)$ is the Heaviside function, $z_{-1}=-\infty$, and $z_{6}=\infty$. A flux of monoenergetic electrons, which do not interact with one another, with the energy $E$ and the concentration $n_{0}$ is assumed to fall on the 3BRTS from the left normally to its planes. Then the wave function $\Psi(z, t)$ of an electron, the motion of which is one-dimensional $\left(\mathbf{k}_{\|}=0\right)$, satisfies the full Schrödinger equation

$i \hbar \frac{\partial \Psi(z, t)}{\partial t}=\left(H_{0}(z)+H(z, t)\right) \Psi(z, t)$.

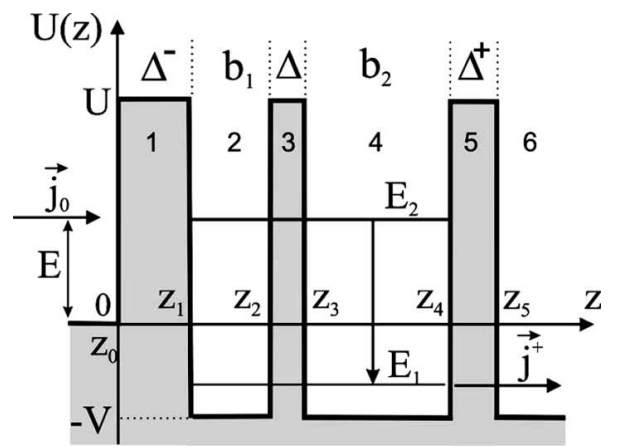

Fig. 1. Energy diagram for of an electron in the 3BRTS with shifted bottoms of potential wells

Here,

$H_{0}(z)=-\frac{\hbar^{2}}{2} \frac{\partial}{\partial z} \frac{1}{m(z)} \frac{\partial}{\partial z}+U(z)$

is the electron Hamiltonian for the stationary Schrödinger problem. Its solution, $\Psi_{0}(z)$, is known [7-9] and enables the exact analytical calculation of the density distribution of a probability to find an electron in the 3BRTS to be carried out:

$W(E)=\frac{1}{z_{5}} \int_{0}^{z_{5}}\left|\Psi_{0}(z)\right|^{2} d z$.

Unlike the transmission factor, the function $W$ governs the spectral characteristics (resonance energies and widths) for quasistationary electron states in the 3BRTSs with arbitrary geometrical parameters and in an arbitrary energy interval [7].

The second term in Eq. (3),

$$
\begin{aligned}
& H(z, t)=-e \mathcal{E}\left[z \theta(z)+\left(z_{5}-z\right) \theta\left(z-z_{5}\right)\right] \times \\
& \times\left(e^{i \omega t}+e^{-i \omega t}\right)
\end{aligned}
$$

is the interaction Hamiltonian for an electron and the time-dependent electromagnetic field with frequency $\omega$ and the amplitude of the electric field strength $\mathcal{E}$.

Under the action of the electromagnetic field, the electron quantum-mechanical transitions between the neighbor quasistationary states with energy absorption or emission take place. Therefore, the solution of the Schrödinger equation (3) in the weak-signal approximation [8-10] and the first order of perturbation theory is sought in the form

$\Psi(z, t)=\sum_{n=-1}^{+1} \Psi_{n}(z) e^{-i\left(\omega_{0}=+n \omega\right) t} \quad\left(\omega_{0}=E / \hbar\right)$. 
Keeping the quantities of the first order of smallness in Eq. (3), the following system of inhomogeneous equations is obtained for the determination of the functions $\Psi_{ \pm 1}(z)$ :

$$
\begin{aligned}
& \left(H_{0}(z)-\hbar\left(\omega_{0} \pm \omega\right)\right) \Psi_{ \pm 1}(z)- \\
& -e \mathcal{E}\left[z \theta(z)+\left(z_{5}-z\right) \theta\left(z-z_{5}\right)\right] \Psi_{0}(z)=0,
\end{aligned}
$$

the solution of which is a superposition of the functions

$\Psi_{ \pm 1}(z)=\Psi_{ \pm}(z)+\Phi_{ \pm}(z)$.

The solutions $\Psi_{ \pm}(z)$ of the homogeneous equations (8) are sought in the form

$$
\begin{aligned}
& \Psi_{ \pm}(z)=\sum_{p=0}^{6}\left[B_{ \pm}^{(p)} e^{-i k_{ \pm}^{(p)} z}+A_{ \pm}^{(p)} e^{i k_{ \pm}^{p} z}\right] \times \\
& \times\left[\theta\left(z-z_{p-1}\right)-\theta\left(z-z_{p}\right)\right],\left(A_{ \pm}^{(0)}=B_{ \pm}^{(6)}=0\right),
\end{aligned}
$$

where

$k_{ \pm}^{(0)}=\sqrt{2 m_{0}(E \pm \hbar \omega) / \hbar^{2}}$,

$k_{ \pm}^{(2,4,6)}=\sqrt{2 m_{2}(E+V \pm \hbar \omega) / \hbar^{2}}$,

$k_{ \pm}^{(1,3,5)}=\sqrt{2 m_{1}((E-U) \pm \hbar \omega) / \hbar^{2}}$.

The exact partial solutions $\Phi_{ \pm}(z)$ of the system of inhomogeneous equations (8) are known $[8,9]$. They are

$$
\begin{aligned}
& \Phi_{ \pm}(z)=\sum_{p=1}^{5}\left[\mp \frac{e \mathcal{E} z}{\hbar \omega} \Psi_{0}^{(p)}(z)+\frac{e \mathcal{E}}{m_{p}(z) \omega^{2}} \frac{d \Psi_{0}^{(p)}(z)}{d z}\right] \times \\
& \times\left[\theta\left(z-z_{p-1}\right)-\theta\left(z-z_{p}\right)\right] \mp \frac{e \mathcal{E} z_{5}}{\hbar \omega} \Psi_{0}^{(6)}\left(z_{5}\right) \theta\left(z-z_{5}\right) .
\end{aligned}
$$

All unknown coefficients $A_{ \pm}^{(p)}$ and $B_{ \pm}^{(p)}$ of the wave function $\Psi_{ \pm}(z)$ (Eq. (10)) are determined from the conditions of continuity for the function and the corresponding fluxes of probability density across all heterointerfaces in the nanosystem. As a result, we obtain the unambiguous full wave function $\Psi(E, z)$, which determines, according to the quantum-mechanical postulates [11], the current density through the nanostructure. Since the size of
3BRTS is small in comparison with the electromagnetic wave length, we use the quasiclassical approximation [8-10] in order to calculate the reduced current density, which is related to the real part of the active dynamic conductivity of the nanosystem,

$\sigma(E, \omega)=\sigma^{+}(E, \omega)+\sigma^{-}(E, \omega)$,

where

$\begin{aligned} \sigma^{+}(E, \omega) & =\frac{\hbar^{2} \omega n_{0}}{2 m_{2} z_{5} \mathcal{E}^{2}}\left(k_{+}^{(6)}\left|A_{+}^{(6)}\right|^{2}-k_{-}^{(6)}\left|A_{-}^{(6)}\right|^{2}\right), \\ \sigma^{-}(E, \omega) & =\frac{\hbar^{2} \omega n_{0}}{2 m_{0} z_{5} \mathcal{E}^{2}}\left(k_{+}^{(0)}\left|B_{+}^{(0)}\right|^{2}-k_{-}^{(0)}\left|B_{-}^{(0)}\right|^{2}\right) .\end{aligned}$

Here, $\sigma^{+}(E, \omega)$ and $\sigma^{-}(E, \omega)$ are the partial components of the dynamic conductivity formed by the fluxes of electrons, which, after their interaction with the electromagnetic field, quit the nanosystem in either the forward $\left(j^{+} \sim \sigma^{+}\right)$or backward $\left(j^{-} \sim \sigma^{-}\right)$ direction with respect to that of the electron flux $j_{0}$ with the concentration $n_{0}$ that falls on the 3BRTS.

\section{Analysis and Discussion of Results}

It is known that the physical parameters of QCLs and QCDs are governed, to a great extent, by the characteristics of RTS, which is an active element of those nanodevices. In their turn, RTSs are characterized by the active dynamic conductivity, $\sigma$, and parameters that are connected with the spectral characteristics - the resonance energies $E_{n}$ and widths $\Gamma_{n}$ - of those quasistationary states, which are engaged into the quantum transition $[9,12]$.

Calculations were carried out, and the obtained results were analyzed for, as an example, an experimentally created and often studied $[2-4,13,14]$ threebarrier nanosystem composed of $\mathrm{In}_{0.52} \mathrm{Al}_{0.48} \mathrm{As}$ potential barriers and $\operatorname{In}_{1-x} \mathrm{Ga}_{x}$ As potential wells. A change of the Ga content $x$ shifts the bottom of the corresponding well, $V=(390-830 x) \mathrm{meV}$, and modifies the effective electron mass in it, $m=(0.067-$ $-0.045 x) m_{e}$. The height of potential barriers with respect to the bottom of the external input potential well $\left(\mathrm{In}_{0.53} \mathrm{Ga}_{0.47} \mathrm{As}\right)$ amounted to $U=516 \mathrm{meV}$. The concentration of electrons in the monoenergetic flux, $n_{0}=10^{16} \mathrm{~cm}^{-3}$, was so selected that the interaction between electrons could be neglected.

ISSN 2071-0194. Ukr. J. Phys. 2013. Vol. 58, No. 2 

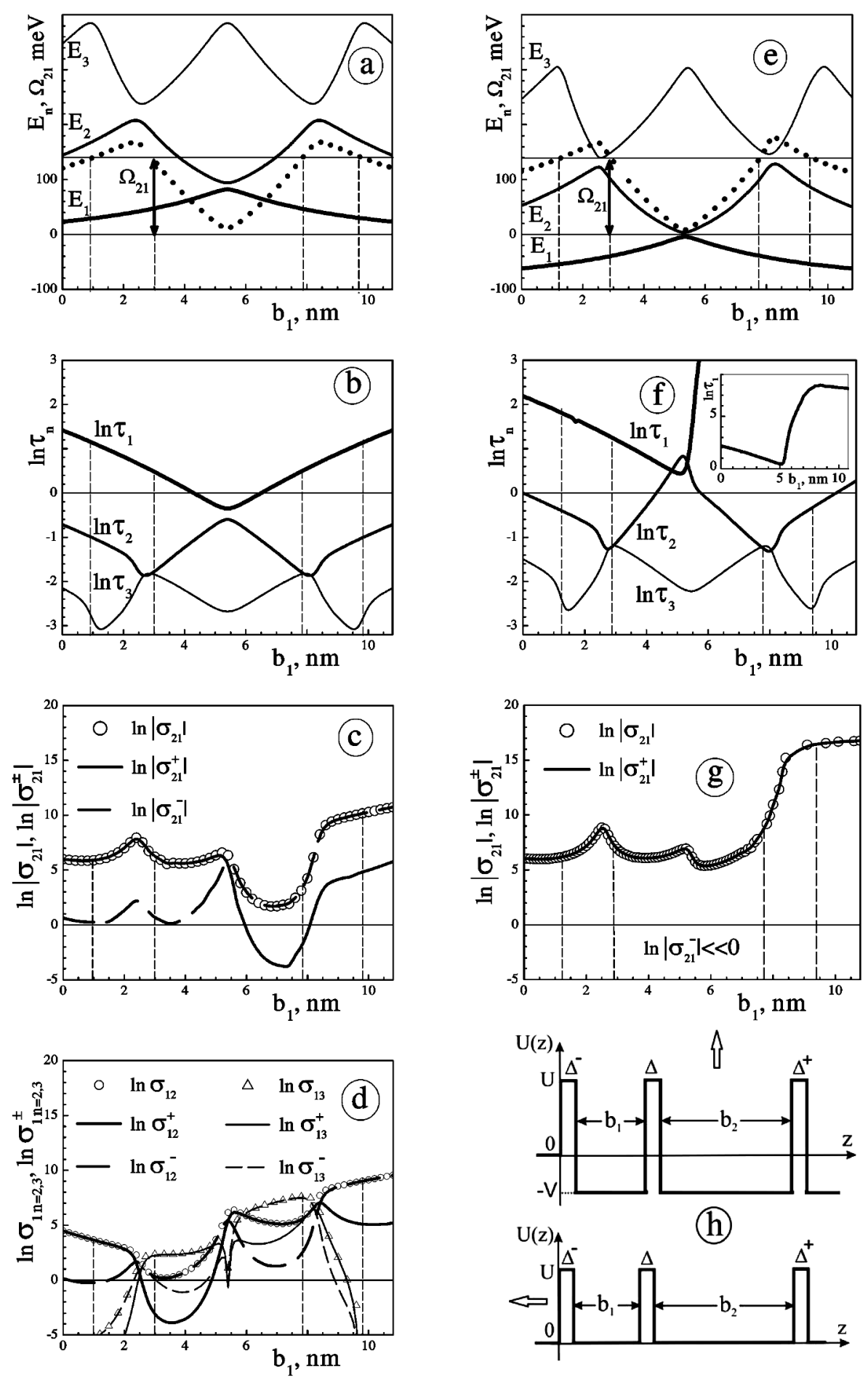

Fig. 2. Dependences of $E_{n}, \ln \tau_{n}, \ln \sigma_{n, m}$, and $\ln \sigma_{n, m}^{ \pm}$on $b_{1}$ for 3 BRTSs at $V=0(a-d)$ and $100 \mathrm{meV}(e-g)$, and $(h)$ the energy diagrams of those systems 
The main properties of the resonance energies, $E_{n}$, lifetimes, $\tau_{n}$, and maximum magnitudes of the active dynamic conductivity, $\sigma$, are illustrated in Fig. 2 using 3BRTSs with identical barrier thicknesses without (Figs. 2, $a$ to $d$ ) and with (Figs. 2,e to $g$ ) shifted bottoms of potential wells as examples. Changes that arise in 3BRTSs with different barrier thicknesses will be discussed after the analysis of a nanosystem with identical barrier thicknesses.

The calculations were carried out for the values $\Delta^{-}=\Delta=\Delta^{+}=2.4 \mathrm{~nm}, b=b_{1}+b_{2}=10.8 \mathrm{~nm}$, $x_{0}=x_{2}=x_{4}=x_{6}=0.47$ (Figs. 2, $a$ to $d$ ) and $x_{0}=0.47, x_{2}=x_{4}=x_{6}=0.35(V=100 \mathrm{meV}$, Figs. 2, $e$ to $g$ ).

In Figs. 2, $a$ and $b$, the calculated dependences of the resonance energies $E_{n}$ and the logarithms of the resonance widths, $\ln \tau_{n}$, for three first QSESs, the transitions between which occur either with emission (a laser, $\sigma_{21} \leq 0$ ) or absorption (a detector, $\sigma_{12}>0$ and $\sigma_{13}>0$ ) of electromagnetic waves, are depicted. In Figs. 2,c and $d$, the same dependences but for the logarithms of the maximum dynamic conductivities are shown.

It is worth noticing that since a reduction of the $\mathrm{Ga}$ content $x$ in the wells shifts the electron spectrum toward negative energies $(E<0)$, we, for the sake of generality, calculated the resonance energies and lifetimes, by using the method of distribution function $W$ [7] rather than the transmission factor $D$, which is inapplicable in the case $E<0$ (for the detector transitions from the first QSES).

The main properties of resonance energies and widths and their relation to the parameters of active conductivity $\sigma(E, \omega)$ were analyzed in work [9] in detail, with a 3BRTS with identical bottoms of potential wells serving an example. In this work, we focus our attention only on new important circumstances that emerge owing to different shifts of well bottoms in laser transitions. We also compare the properties of conductivity in the 3BRTS that arise in the case where an electromagnetic field is absorbed or emitted.

From Figs. 2, $a, b, e$, and $f$, one can see that a change in the position $b_{1}$ of the internal barrier between the external ones changes the values of resonance energies $E_{n}$ and the logarithms of lifetimes $\ln \tau_{n}$ so that, provided identical values for the bottoms of all wells and the external barriers, the magnitudes of $\ln \tau_{n}$ decrease proportionally to $E_{n}$. In so doing, both $E_{n}$ and $\ln \tau_{n}$ are symmetric functions with respect to the middle point $\left(b_{1}=b / 2\right)$ of the common potential well of the width $b$.

A reduction of the Ga content $x$ in the output and both internal wells (Fig. 2,e) shifts their bottoms toward negative energies, so that the whole spectrum of resonance energies also shifts in the same direction, and the lowest resonance energies enter the range $E<0$ one by one. It is important to notice that the negative shift of well bottoms does not substantially affect the shape of the dependences of all resonance energies on $b_{1}$. It reduces their values and, accordingly, increases the values of $\ln \tau_{n}$ for those QSESs, the resonance energies of which satisfy the condition $E_{n}>0$. However, if the QSESs enter the region $E<0$, the dependences of the corresponding $\ln \tau_{n}$ on $b_{1}$ change very much.

For instance, from Figs. 2,e and $f$, one can see that, since $E_{2}>0$ and $E_{3}>0$, the shapes of the dependences of $\ln \tau_{2}$ and $\ln \tau_{3}$ on $b_{1}$ change weakly, whereas that of $\ln \tau_{1}$ varies very substantially; in the interval $0 \leq b_{1} \leq b / 2$, the quantity $\ln \tau_{1}$ weakly increases, and, at $b / 2 \leq b_{1} \leq b$, it drastically grows. Such a behavior of $\ln \tau_{1}$, which considerably affects the dynamic conductivity of RTS, can be understood from the physical reasoning. Really, as far as the internal barrier is in the left half-space between the external barriers, the electron in the first QSES mainly dwells in the right wider well and penetrates from there through the right barrier into the right external well. Therefore, at $0 \leq b_{1} \leq b / 2$, the value of $\ln \tau_{1}$ grows only slightly in comparison with the case of 3BRTS with identical bottoms in all wells (Fig. 2,b). However, if the internal barrier is in the right half-space between the external barriers, the electron in the first quasistationary state dwells with a higher probability in the left wider well; and it is hard for it to tunnel to the input, because the input medium creates a low, but infinitely wide, potential barrier for it (Fig. 1). Two (rather than one) barriers-the internal and external ones-should be overcome on the way to the exit through the RTS. As a result, the lifetime $\tau_{1}$ in the first QSES in the interval $b / 2 \leq b_{1} \leq b$ drastically grows with $b_{1}$.

The revealed properties of $E_{n}$ and $\tau_{n}$ are responsible for the behavior of dynamic conductivity in the 3BRTS depending on the position of the internal barrier with respect to the external ones.

ISSN 2071-0194. Ukr. J. Phys. 2013. Vol. 58, No. 2 
To optimize the functioning of 3BRTS as an active element of QCL or QCD, the maximum conductivity $\sigma$ has to be provided at a given (required) energy of emitted or absorbed fields $(\Omega=\hbar \omega)$ and at the values of both the input flux $\left(j_{0} \sim \sqrt{E}\right)$ and the larger of the lifetimes $\left(\tau_{n}\right)$ of working QSESs as minimum as possible.

Let us analyze firstly the $b_{1}$-dependence of the maximum value of negative conductivity $\sigma_{21}=$ $=\max |\sigma(E, \Omega)|$ that arises at the laser transition, at which an electron from the monoenergetic flux emitted by the injector transits onto the second QSES, emits a quantum of the electromagnetic field with the energy $\Omega_{21}=\hbar \omega_{21}=E_{2}-E_{1}$, transits onto the first QSES, and tunnels further through the 3BRTS. From Fig. 2,c and $g$, one can see that, depending on the position $b_{1}$ of the internal barrier, the dynamic conductivities $\sigma_{21}$ 's in 3BRTSs with shifted (Fig. 2,g) and identical (Fig. 2,c) bottoms of the wells are formed in essentially different ways by their partial components $\sigma_{21}^{ \pm}$. In the 3BRTS with identical well bottoms (Fig. 2,c), the maximum of conductivity $\sigma_{21}$ at $0 \leq b_{1} \leq b / 2$ is mainly formed by the forward flux $\left(\sigma_{21}^{+} \gg \sigma_{21}^{-}\right)$. At $b_{1} \approx b / 2$, the fluxes in both directions are almost equal to each other $\left(\sigma_{21}^{+} \approx \sigma_{21}^{-}\right)$, and, at $b / 2 \leq b_{1} \leq b$, the backward flux prevails $\left(\sigma_{21}^{-} \gg \sigma_{21}^{+}\right)$. In the 3BRTS with the shifted bottoms of the output external and internal wells (Fig. 2,g), the conductivity $\sigma_{21}$ is formed only by the forward flux, irrespective of the internal barrier position $\left(0 \leq b_{1} \leq b\right)$, because $\sigma_{21}^{+} \gg \sigma_{21}^{-}$.

Let us determine now which of 3BRTS configurations are optimum for the functioning of QCLs. Let a nanolaser emit an electromagnetic field with the energy $\Omega_{21}=E_{2}-E_{1}$. As is seen from Figs. 2,a and $e$, it can be provided by the quantum transition between the second and first QSESs in both nanosystems at four geometrical configurations of 3BRTSs. From Fig. 2, one can see that the configurations, in which the internal barrier is located in the right half of the well are bad, because, in the case of identical depths of all RTS wells (Fig. 2,c), the conductivity $\sigma_{21}$ is formed by the harmful backward flux $\left(\sigma_{21}^{-} \gg \sigma_{21}^{+}\right)$. At the same time, although $\sigma_{21}^{+} \gg \sigma_{21}^{-}$ for shifted well bottoms (Fig. 2,g), the lifetime $\tau_{1}$ is too long, and, accordingly, the dissipative processes (phonons and others) will disturb the laser from the coherent state. Of two good 3BRTS configurations (at $0 \leq b_{1} \leq b / 2$ ) for both nanosystems, optimum are those, in which the internal barrier is located in the second quarter of the common well, because, at almost equal $\sigma_{21}$-values, the lifetime in them is much shorter, so that influence of dissipative processes is minimum.

A comparison between the optimum 3BRTS configurations with the identical and shifted bottoms of the wells shows that, in the former, the lifetime $\tau_{1}$ is shorter, but the excitation current $\left(j_{0} \sim \sqrt{E_{2}}\right)$ is higher than that in the latter (Figs. 2,e and $f$ ). Hence, a reduction of the Ga content $x$ in the output and internal wells favors a reduction of the excitation current magnitude, although the negative influence of dissipative processes becomes a little stronger at that.

Now, let us analyze the functioning of 3BRTS as an active element of QCD that works at quantum transitions onto higher QSESs from the first one, which is supplied with the monoenergetic electron flux from the extractor. Unlike the laser regime, in the processes of electromagnetic wave absorption by electrons passing through the RTS, the dynamic conductivity of a detector can be formed at both $1 \rightarrow 2$ and $1 \rightarrow 3$ quantum transitions, depending on the position of the internal barrier between the external ones.

From Fig. 2,d, one can see that the 3BRTS configuration with constant bottoms of all wells is not efficient again if the internal barrier is located in the right half of the common well $\left(b / 2 \leq b_{1} \leq b\right)$, because, here, in the third quarter, $\sigma_{13} \gg \sigma_{12}$, but $\sigma_{13}^{-} \gg \sigma_{13}^{+}$, whereas in the fourth quarter, $\sigma_{12} \gg \sigma_{13}$, but $\sigma_{12}^{-} \gg \sigma_{12}^{+}$. Therefore, this 3BRTS functions optimally at the quantum transitions $1 \rightarrow 2$, when the internal barrier is in the first quarter of the common well, or at the transitions $1 \rightarrow 3$, when it is in the second quarter of the common well. From Fig. 2, $d$, one can see that, at the transition $1 \rightarrow 2$, the absorbed field energy $\Omega_{12}$ is lower than the energy $\Omega_{13}$ absorbed at the transition $1 \rightarrow 3$. In this case, the maximum of the conductivity $\sigma_{12}$ by a little larger than $\sigma_{13}$, because the lifetime $\tau_{1}$ is longer if the internal barrier is located in the first rather than the second quarter.

It is worth noticing that, as is seen from Fig. 2, $d$, the arrangement of the internal barrier near the position $b_{1} \approx b / 4$ is inexpedient, because here $\sigma_{12} \approx \sigma_{13}$, $\sigma_{12}^{+} \approx \sigma_{12}^{-}$, and $\sigma_{13}^{+} \approx \sigma_{12}^{-}$.

The corresponding calculations and analysis brought about the following results. If the total thick- 
ness of all barriers $\left(\Delta^{-}+\Delta+\Delta^{+}\right)$in optimum 3BRTS configurations with an arbitrary shift of well bottoms is fixed, the system with the identical thicknesses of the external barriers is the best, because a reduction in the thickness of the input barrier gives rise to an increase of the electron lifetime in functioning QSESs, but does not substantially change the magnitude of $\sigma$. At the same time, an increase of this barrier thickness weakly diminishes the lifetime and considerably decreases the magnitude of $\sigma$. On the other hand, an increase in the thickness of the internal barrier, provided the corresponding reduction of the thicknesses of the external ones, exponentially reduces both the lifetime and the magnitude of $\sigma$.

\section{Conclusions}

The theory of spectral parameters $E_{n}$ and $\Gamma_{n}$ and the active dynamic conductivity $\sigma$ of electrons interacting with an electromagnetic field in open 3BRTSs with arbitrary physical and geometrical parameters has been developed. It is shown that the choice of physical parameters and a geometrical design of RTS allows one to optimize its functioning as an active element in QCLs or QCDs.

1. A.F. Kazarinov and R.A. Suris, Fiz. Tekh. Poluprovodn. 6, 135 (1972).

2. J. Faist, F. Capasso, D.L. Sivco, C. Sirtori, A.L. Hutchinson, and A.Y. Cho, Science 264, 553 (1994).

3. J. Faist, F. Capasso, and C. Sirtori, Appl. Phys. Lett. 66, 538 (1995).

4. C. Gmachl, F. Capasso, D.L. Sivco, and A.Y. Cho, Rep. Prog. Phys. 64, 1533 (2001).

5. F.R. Giorgetta, E. Baumann, D. Hofstetter, C. Manz, Q. Yang, K. Köhler, and M. Graf, Appl. Phys. Lett. 91, 111115 (2007).

6. V. Berger, Patent France No. 0109754 (2001).
7. N.V. Tkach and Yu.A. Seti, Fiz. Nizk. Temp. 35, 710 (2009).

8. M.V. Tkach and Yu.O. Seti, Ukr. Fiz. Zh. 55, 801 (2010).

9. N.V. Tkach and Yu.A. Seti, Fiz. Tekh. Poluprovodn. 45, 387 (2011).

10. E.I. Golant, A.B. Pashkovskii, and A.S. Tager, Fiz. Tekh. Poluprovodn. 28, 740 (1994).

11. L.D. Landau and E.M. Lifshitz, Quantum Mechanics. Non-Relativistic Theory (Pergamon Press, New York, 1977).

12. M.V. Tkach, O.M. Makhanets, Ju.O. Seti, M.M. Dovganiuk, and O.M. Voitsekhivska, Acta Phys. Pol. A 117, 965 (2010).

13. Q.J. Wang, C. Pflug, L. Diehl, F. Capasso, T. Edamura, S. Furuta, M. Yamanishi, and H. Kan, Appl. Phys. Lett. 94, 011103 (2009).

14. D. Hofstetter, F.R. Giorgetta, E. Baumann, Q. Yang, C. Manz, and K. Köhler, Appl. Phys. Lett. 93, 221106 (2008).

Received 28.08.12.

Translated from Ukrainian by O.I. Voitenko

M.B. Ткач, Ю.О. Cemi

ТЕОРІЯ ВЛАСТИВОСТЕЙ

РЕЗОНАНСНО-ТУНЕЛЬНИХ НАНОСТРУКТУР, ЯК АКТИВНИХ ЕЛЕМЕНТІВ КВАНТОВИХ

КАСКАДНИХ ЛАЗЕРІВ І ДЕТЕКТОРІВ

Р е з ю м е

На основі моделі прямокутних потенціалів та ефективних мас електрона побудована теорія спектральних параметрів квазістаціонарних станів та динамічної провідності відкритої трибар'єрної резонансно-тунельної системи, як активного елемента квантового каскадного лазера чи квантового каскадного детектора. Показано, що оптимальна робота трибар'єрної резонансно-тунельної структури досягається належним вибором іiї геометричної конфігурації, за рахунок розміщення внутрішнього бар'єра ближче до вхідного в такому положенні, яке визначається величиною енергії електромагнітного поля. 